\title{
Study the Profile of the SHGS Members Engaged in Agriculture and Allied Enterprises
}

\author{
Ramadevi G. Nair ${ }^{*}$, R. P. Kadam and A. S. Lad \\ Department of Extension Education, Vasantrao Naik Marathwada Krishi Vidyapeeth, \\ Parbhani (M.S.) India \\ *Corresponding author
}

\section{A B S T R A C T}

\section{Keywords}

Self help groups (SHGs), SHGs members and Profile

\section{Article Info}

Accepted:

28 January 2021

Available Online:

10 February 2021
The present study was conducted purposively in Nanded and Parbhani districts of the Marathwada region of Maharashtra state during the year 2019-20 in jurisdiction of Mahila Arthic Vikas Mahamandal Organization (MAVIM) and District Rural Development Agency (DRDA) in Marathwada region of Maharashtra state as significant work done by both MAVIM and DRDA in the formation of SHGs. The main aim is to study the profile of the SHGs members engaged in agriculture and allied enterprises. The following independent variables were selected for the study viz, age, education, family type, caste, annual income, occupation, land holding, experience in SHGs enterprise, information seeking behaviour, institutional training received, source of motivation and socioeconomic impact of microfinance on the members of self help groups in Marathwada region of Maharashtra State was considered as dependent variable based on the objectives set forth for the study.

\section{Introduction}

In the words of Mahatma Gandhi, "India Lives in Villages". Rural population in India was reported at $65.53 \%$ in 2019 , according to World Bank collection of development indicators compiled from officially recognized sources. It is the reason why the Indian Government has always focused on the development of the rural areas of the country by various welfare programmes meant for the rural people. In the rural areas, the women constitute a huge workforce and contribute significantly towards agriculture and labour force. Despite all these, the condition of women in rural areas remains poor and demands concern. In recent years, SHG movement has gained momentum in a national level, has helped women from far corners of rural area to generate income of their own, mobilize them through groups and gave voice to their opinions.

Self-help groups (SHGs) are small groups of people facing similar problems. The members of the group help each other to solve their problems. A reasonably educated but helpful local person takes the lead in mobilizing these people to form a group. The person called animator or facilitator helps the group 
members develop the habit of thrift and promote small savings among them. The group savings are kept in a common bank account from which small loans are given to members. After six months, the SHG can approach any bank for availing loan facility to undertake a suitable entrepreneurial activity. The group loan is distributed among the members to run a small business. The loan is repaid out of the profits earned (Lathkar Malhar, 2009)

The rural poor women especially from BPL families are being focused and encouraged to form groups preferably with members of same socio economic background and carryout thrift and credit activity. Later they are trained and encouraged to take loan from banks to carryout entrepreneurial activities to generate income of their own. The simple yet powerful concept of SHG has helped millions of women so far to help them realize their true potential. The origin of the SHG traces back to Bangladesh and Prof. Mohammed Yunus is regarded as the father of SHG movement. In India, the SHG movement gained momentum after SHG bank linkage programme in 1992. The group based self help approach not only helps women to carryout income generating activities, but also helped them to take part in formal banking system thus by improving their self confidence (Sucharita and Bishnoi, 2019).

\section{Materials and Methods}

For the present study out of eight districts of Marathwada region of Maharashtra state two districts were purposively selected i.e. Nanded and Parbhani based on maximum number of self help groups during the years 2019-20 in jurisdiction of Mahila Arthic Vikas Mahamandal Organization (MAVIM) and District Rural Development Agency (DRDA) in Marathwada region of Maharashtra state as significant work done by both MAVIM and DRDA in the formation of SHGs. Three talukas were purposively selected from each district for the present study on the basis of maximum numbers of self help groups. The selected talukas from Parbhani district were Parbhani, Purna and Gangakhed as well as from Nanded district the selected talukas were Kinwat, Nanded and Aardhapur these all talukas were selected on the basis of the maximum numbers of self help groups. From each selected village 07 women members of self help group were selected randomly to make a sample of 35 women members of self help group from each selected talukas and the total 105 women members of self help groups from each district. Thus, total to the tune of 210 women members of self help group were selected for present study from both the districts. Ex-post facto research design was adopted in this study. The data were collected with the help of pretested interview schedule. The statistical methods and tests such as frequency, percentage, mean, standard deviation, coefficient of correlation, multiple regressions, $\mathrm{Z}$ test and path analysis were used for the analysis of data.

\section{Objective}

To study the profile of the SHGs members engaged in agriculture and allied enterprises.

\section{Results and Discussion}

\section{Profile of the SHGs members engaged in agriculture and allied enterprises}

One of the objectives of present study was to study the profile of SHGs members i.e. personal, socio-economic and psychological characteristics of the SHGs members. The study of personal, socio-economical and psychological characteristics of SHGs members were made with reference to age, education, family type, Caste, annual income, 
occupation, land holding, experience in SHGs enterprise, information seeking behaviour, institutional training received and sources of motivation. The SHGs members engaged in different enterprise i.e. Sewing, Processing, Agriculture (Agri.), Animal husbandry and Dairy (AHD), in other business and as a Labour. Data were analyzed in respect to above listed variables and the results are presented here under.

\section{Age}

Age influences the behaviour of an individual by revealing her to varied situation for number of times. It is an important factor influencing attitude of a member through various ways, ultimately affecting managerial ability, skill and judgment required in the performance of SHGs. It was observed that at overall level more than two third 67.14 per cent of the SHGs members belonged to 'middle age' category that is 29 to 47 years and at same time it was also found that majority 79.16 per cent of the SHGs members having middle age group engaged in sewing operation. The findings of the present study are in conformity with the findings of Pattanaik (2009), Bhandari (2014), Adsul (2016), Fazely (2016), Hasan et al., (2016) and Dhulgand (2020) (Table 1).

\section{Education}

Formal education enhances comprehension abilities and skill of an individual. An educated individual is more prone to analyze cause and affect relationship in different aspects of life. This leads in improving their problem solving ability. It was found that $(28.6 \%)$ of the SHGs members were educated up to middle school level i.e. $\left(5^{\text {th }}\right.$ to $7^{\text {th }}$ standard) as well as it was also found that majority 35.00 per cent of the SHGs members took the education up to middle school level were working as labour. The findings of the present study are in conformity with the findings of Krishnakumar (2002), Lairinliana and Kanagaraj (2006) (Table 2).

\section{Family type}

From the table 3 it was revealed that, 73.80 per cent of SHGs members were live in joint family. It was also found that majority 92.60 per cent of the SHGs members of joint family were engaged in processing. This clearly showed that the majority of selected SHGs members belonged to joint families. This might be due to importance attached to kinship as well as their age old dependency on supplementary labour which is making them to give preference to joint families for economic and social security reasons. This result was in agreement with the findings of Jadhav (2018), Raut (2018), Sucharita and Bishnoi (2019), and Dhulgand (2020).

\section{Caste}

Caste determines the role of an individual both as an individual and as a member of society. It is clear from table 4 that, 53.30 per cent of SHGs members belonging to middle caste i.e. (OBC and Muslim). It was also found that majority 58.50 per cent of the SHGs members from OBC and Muslim castes were engaged in agriculture. These observations lead to conclude that maximum number of SHGs members belonged to middle and lower castes. However, the upper caste members were also noticed in a considerable numbers. The policy of the Government regarding uplifting economically weaker sections might be responsible for the higher representation of middle and lower caste in SHGs. Secondly, the classification of the SHGs members might reassemble with the caste structure in the region. The findings were well supported by the research findings of Singh et al., (2001), Rewale (2003) and Topare (2006). 
Table.1 Distribution of the SHGs members according to their age

\begin{tabular}{|c|c|c|c|c|c|}
\hline \multirow[t]{3}{*}{ Sr. No. } & \multirow[t]{3}{*}{ Enterprise } & \multicolumn{3}{|c|}{ Category } & \multirow[t]{3}{*}{ Total $(n=210)$} \\
\hline & & Young & Middle & Old & \\
\hline & & Up to 28 & 29 to 47 & $48 \&$ above & \\
\hline 1. & Sewing & $5(10.42)$ & 38 (79.16) & $5(10.42)$ & $48(100)$ \\
\hline 2. & Processing & $4(14.80)$ & $20(74.10)$ & $3(11.10)$ & $27(100)$ \\
\hline 3. & Agri. & $6(14.60)$ & $26(63.40)$ & $9(22.00)$ & $41(100)$ \\
\hline 4. & AHD & $6(20.70)$ & $19(65.50)$ & $4(13.80)$ & $29(100)$ \\
\hline 5. & Other business & $8(32.00)$ & $8(32.00)$ & $9(36.00)$ & $25(100)$ \\
\hline 6. & Labour & $6(15.00)$ & $30(75.00)$ & $4(10.00)$ & $40(100)$ \\
\hline \multicolumn{2}{|c|}{ Average age (Yrs.) } & 35 (16.67) & $141(67.14)$ & 34 (16.19) & $210(100)$ \\
\hline \multicolumn{4}{|c|}{ Mean $=37.68$} & $. D .=10.31$ & \\
\hline
\end{tabular}

(Figures in the parentheses indicate percentages)

Table.2 Distribution of the SHGs members according to their education

\begin{tabular}{|c|c|c|c|c|c|c|c|c|c|}
\hline \multirow{3}{*}{$\dot{\dot{Z}}$} & \multirow{3}{*}{ 盛 } & \multicolumn{7}{|c|}{ Category } & \multirow{3}{*}{$\begin{array}{l}\stackrel{0}{\pi} \\
\stackrel{\pi}{\pi} \\
\stackrel{\pi}{0} \\
\bullet\end{array}$} \\
\hline & & 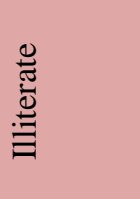 & 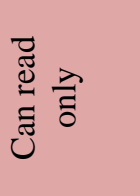 & 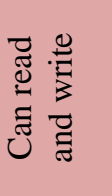 & 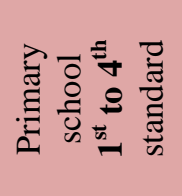 & 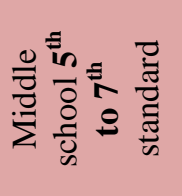 & 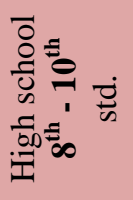 & $\frac{0}{0} \overline{0}$ & \\
\hline & & $\mathbf{1}$ & 2 & 3 & 4 & 5 & 6 & 7 & \\
\hline 1 & 路 & $\begin{array}{c}13 \\
(27.10)\end{array}$ & $\begin{array}{c}00 \\
(00.00)\end{array}$ & $\begin{array}{c}04 \\
(8.30)\end{array}$ & $\begin{array}{c}15 \\
(31.30)\end{array}$ & $\begin{array}{c}08 \\
(16.70)\end{array}$ & $\begin{array}{c}03 \\
(6.30)\end{array}$ & $\begin{array}{c}05 \\
(10.30)\end{array}$ & $\begin{array}{c}48 \\
(100)\end{array}$ \\
\hline 2 & 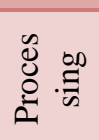 & $\begin{array}{c}7 \\
(25.90)\end{array}$ & $\begin{array}{c}00 \\
(00.00)\end{array}$ & $\begin{array}{c}01 \\
(3.70)\end{array}$ & $\begin{array}{c}06 \\
(22.30)\end{array}$ & $\begin{array}{c}\mathbf{0 8} \\
(\mathbf{2 9 . 6 0})\end{array}$ & $\begin{array}{c}03 \\
(11.10)\end{array}$ & $\begin{array}{c}02 \\
(7.40)\end{array}$ & $\begin{array}{c}27 \\
(100)\end{array}$ \\
\hline 3 & 家 & $\begin{array}{c}09 \\
(21.90)\end{array}$ & $\begin{array}{c}00 \\
(00.00)\end{array}$ & $\begin{array}{c}02 \\
(4.90)\end{array}$ & $\begin{array}{c}09 \\
(21.90)\end{array}$ & $\begin{array}{c}13 \\
(31.70)\end{array}$ & $\begin{array}{c}05 \\
(12.20)\end{array}$ & $\begin{array}{c}03 \\
(7.30)\end{array}$ & $\begin{array}{c}41 \\
(100)\end{array}$ \\
\hline 4 & 王 & $\begin{array}{c}07 \\
(24.10)\end{array}$ & $\begin{array}{c}00 \\
(00.00)\end{array}$ & $\begin{array}{c}02 \\
(6.90)\end{array}$ & $\begin{array}{c}06 \\
(20.70)\end{array}$ & $\begin{array}{c}09 \\
(31.10)\end{array}$ & $\begin{array}{c}03 \\
(10.30)\end{array}$ & $\begin{array}{c}02 \\
(6.90)\end{array}$ & $\begin{array}{c}29 \\
(100)\end{array}$ \\
\hline 5 & 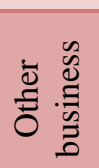 & $\begin{array}{c}07 \\
(28.00)\end{array}$ & $\begin{array}{c}00 \\
(00.00)\end{array}$ & $\begin{array}{c}01 \\
(4.00)\end{array}$ & $\begin{array}{c}05 \\
(20.00)\end{array}$ & $\begin{array}{c}\mathbf{0 8} \\
(\mathbf{3 2 . 0 0})\end{array}$ & $\begin{array}{c}02 \\
(8.00)\end{array}$ & $\begin{array}{c}02 \\
(8.00)\end{array}$ & $\begin{array}{c}25 \\
(100)\end{array}$ \\
\hline 6 & 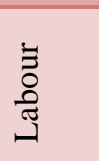 & $\begin{array}{c}10 \\
(25.00)\end{array}$ & $\begin{array}{c}00 \\
(00.00)\end{array}$ & $\begin{array}{c}01 \\
(2.50)\end{array}$ & $\begin{array}{c}09 \\
(22.50)\end{array}$ & $\begin{array}{c}14 \\
(35)\end{array}$ & $\begin{array}{c}06 \\
(15.00)\end{array}$ & $\begin{array}{c}00 \\
(00.00)\end{array}$ & $\begin{array}{c}40 \\
(100)\end{array}$ \\
\hline \multicolumn{2}{|c|}{ 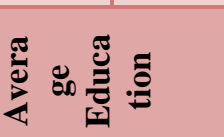 } & $\begin{array}{c}53 \\
(25.20)\end{array}$ & $\begin{array}{c}00 \\
(\mathbf{0 0 . 0 0})\end{array}$ & $\begin{array}{c}11 \\
(5.20)\end{array}$ & $\begin{array}{c}50 \\
(23.80)\end{array}$ & $\begin{array}{c}60 \\
(28.60)\end{array}$ & $\begin{array}{c}22 \\
(10.50)\end{array}$ & $\begin{array}{c}14 \\
(6.70)\end{array}$ & $\begin{array}{c}210 \\
(100)\end{array}$ \\
\hline
\end{tabular}

(Figures in the parentheses indicate percentages) 
Table.3 Distribution of the SHGs members according to their family type

\begin{tabular}{|r|l|c|c|c|}
\hline \multirow{2}{*}{ Sr. No. } & \multirow{2}{*}{ Enterprise } & \multicolumn{2}{|c|}{ Category } & \multirow{2}{*}{ Total (n=210) } \\
\cline { 3 - 4 } & & Joint & Nuclear & \\
\cline { 3 - 4 } & & $\mathbf{2}$ & $\mathbf{1}$ & \\
\hline $\mathbf{1}$ & Sewing & $\mathbf{3 6}(\mathbf{7 5 . 0 0})$ & $12(25.00)$ & $\mathbf{4 8}(\mathbf{1 0 0})$ \\
\hline $\mathbf{2}$ & Processing & $\mathbf{2 5 ( 9 2 . 6 0 )}$ & $02(7.40)$ & $\mathbf{2 7}(\mathbf{1 0 0})$ \\
\hline $\mathbf{3}$ & Agri. & $\mathbf{2 8}(\mathbf{6 8 . 3 0})$ & $13(31.70)$ & $\mathbf{4 1}(\mathbf{1 0 0})$ \\
\hline $\mathbf{4}$ & AHD & $\mathbf{2 2}(\mathbf{7 5 . 9 0})$ & $07(24.10)$ & $\mathbf{2 9}(\mathbf{1 0 0})$ \\
\hline $\mathbf{5}$ & Other business & $\mathbf{2 1}(\mathbf{8 4 . 0 0})$ & $04(16.00)$ & $\mathbf{2 5}(\mathbf{1 0 0})$ \\
\hline $\mathbf{6}$ & Labour & $\mathbf{2 3}(\mathbf{5 7 . 5 0})$ & $17(42.50)$ & $\mathbf{4 0}(\mathbf{1 0 0})$ \\
\hline \multicolumn{2}{|}{ Average family type } & $\mathbf{1 5 5}(\mathbf{7 3 . 8 0})$ & $\mathbf{5 5}(\mathbf{2 6 . 2 0})$ & $\mathbf{2 1 0}(\mathbf{1 0 0})$ \\
\hline
\end{tabular}

(Figures in parentheses indicate percentages)

Table.4 Distribution of the SHGs members according to their caste

\begin{tabular}{|c|c|c|c|c|c|}
\hline \multirow[t]{3}{*}{ Sr. No. } & \multirow[t]{3}{*}{ Enterprise } & \multicolumn{3}{|c|}{ Category } & \multirow{3}{*}{$\begin{array}{c}\text { Total } \\
(\mathbf{n}=210)\end{array}$} \\
\hline & & Lower & Middle & Upper & \\
\hline & & Up to 2 & 3 & $4 \&$ above & \\
\hline 1 & Sewing & $14(29.20)$ & $28(58.30)$ & $6(12.50)$ & $48(100)$ \\
\hline 2 & Processing & $8(29.60)$ & $11(40.80)$ & $8(29.60)$ & $27(100)$ \\
\hline 3 & Agri. & $8(19.50)$ & $24(58.50)$ & $9(22.00)$ & $41(100)$ \\
\hline 4 & AHD & $8(27.60)$ & $15(51.70)$ & $6(20.70)$ & $29(100)$ \\
\hline 5 & Other business & $6(24.00)$ & $14(56.00)$ & $5(20.00)$ & $25(100)$ \\
\hline 6 & Labour & $12(30.00)$ & $20(50.00)$ & $8(20.00)$ & $40(100)$ \\
\hline \multicolumn{2}{|c|}{ Average caste } & $56(26.70)$ & $112(53.30)$ & $42(20.00)$ & $210(100)$ \\
\hline \multicolumn{4}{|c|}{ Mean $=2.56$} & S.D. $=1.20$ & \\
\hline
\end{tabular}

(Figures in the parentheses indicate percentages)

Table.5 Distribution of the SHGs members according to their annual income

\begin{tabular}{|c|c|c|c|c|c|}
\hline \multirow{3}{*}{$\begin{array}{l}\text { Sr. } \\
\text { No. }\end{array}$} & \multirow[t]{3}{*}{ Enterprise } & \multicolumn{3}{|c|}{ Category } & \multirow{3}{*}{$\begin{array}{c}\text { Total } \\
(\mathbf{n}=\mathbf{2 1 0})\end{array}$} \\
\hline & & Low & Medium & High & \\
\hline & & Up to 25218 & 25219- 57123 & $\begin{array}{c}57124 \& \\
\text { above }\end{array}$ & \\
\hline 1 & Sewing & $1(2.00)$ & 44 (91.70) & $3(6.30)$ & $48(100)$ \\
\hline 2 & Processing & $2(7.40)$ & 21 (77.80) & $4(14.80)$ & $27(100)$ \\
\hline 3 & Agri. & $3(7.30)$ & 32 (78.10) & $6(14.60)$ & $41(100)$ \\
\hline 4 & AHD & $6(20.70)$ & $15(51.70)$ & $8(27.60)$ & $29(100)$ \\
\hline 5 & Other business & $2(8.00)$ & $16(64.00)$ & $7(28.00)$ & $25(100)$ \\
\hline 6 & Labour & $22(55.00)$ & $15(37.50)$ & $3(75.00)$ & $40(100)$ \\
\hline \multicolumn{2}{|c|}{ Average income } & $36(17.7)$ & $143(68.10)$ & $31(14.80)$ & $210(100)$ \\
\hline & \multicolumn{3}{|c|}{ Mean $=41171.42$} & \multicolumn{2}{|c|}{ S.D. $=15952.65$} \\
\hline
\end{tabular}

(Figures in the parentheses indicate percentages) 
Table.6 Distribution of the SHGs members according to their occupation

\begin{tabular}{|c|l|c|c|c|}
\hline \multirow{2}{*}{ Sr. No. } & \multirow{2}{*}{ Enterprise } & \multicolumn{2}{|c|}{ Occupation } & \multirow{2}{*}{ Total $(\mathbf{n = 2 1 0})$} \\
\cline { 3 - 4 } & & Main & Main+Subsidiary & \multirow{2}{*}{} \\
\cline { 3 - 4 } & & $\mathbf{2}$ & $\mathbf{1}$ & \\
\hline $\mathbf{1}$ & Sewing & $7(14.60)$ & $\mathbf{4 1}(\mathbf{8 5 . 4 0})$ & $\mathbf{4 8}(\mathbf{1 0 0})$ \\
\hline $\mathbf{2}$ & Processing & $5(18.50)$ & $\mathbf{2 2}(\mathbf{8 1 . 5 0})$ & $\mathbf{2 7}(\mathbf{1 0 0})$ \\
\hline $\mathbf{3}$ & Agri. & $\mathbf{3 2}(\mathbf{7 8 . 1 0})$ & $9(21.90)$ & $\mathbf{4 1}(\mathbf{1 0 0})$ \\
\hline $\mathbf{4}$ & AHD & $10(34.50)$ & $\mathbf{1 9}(\mathbf{6 5 . 5 0})$ & $\mathbf{2 9}(\mathbf{1 0 0})$ \\
\hline $\mathbf{5}$ & Other business & $3(12.00)$ & $\mathbf{2 2}(\mathbf{8 8 . 0 0})$ & $\mathbf{2 5}(\mathbf{1 0 0})$ \\
\hline $\mathbf{6}$ & Labour & $\mathbf{2 6}(\mathbf{6 5 . 0 0})$ & $14(35.00)$ & $\mathbf{4 0}(\mathbf{1 0 0})$ \\
\hline \multicolumn{2}{|c|}{ Average Occupation } & $\mathbf{8 3 ( 3 9 . 5 0 )}$ & $\mathbf{1 2 7}(\mathbf{6 5 . 5 0})$ & $\mathbf{2 1 0}(\mathbf{1 0 0})$ \\
\hline
\end{tabular}

(Figure in the parentheses indicate percentages)

Table.7 Distribution of the SHGs members according to their land holding

\begin{tabular}{|c|c|c|c|c|c|c|c|c|}
\hline \multirow{3}{*}{$\begin{array}{l}\text { Sr. } \\
\text { No. }\end{array}$} & \multirow{3}{*}{ 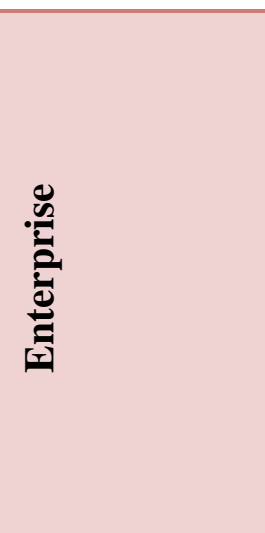 } & \multicolumn{6}{|c|}{ Category } & \multirow{3}{*}{ 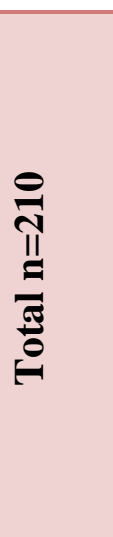 } \\
\hline & & 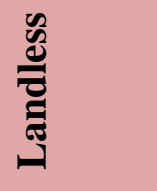 & 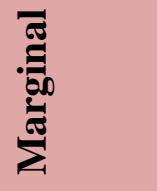 & 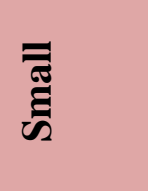 & 苞 & 拝 & 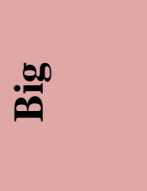 & \\
\hline & & $\frac{\bar{\Xi}}{\frac{\pi}{\sigma}}$ & 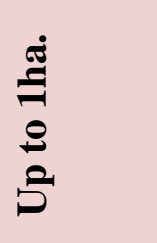 & 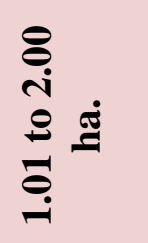 & 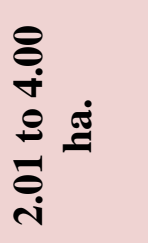 & 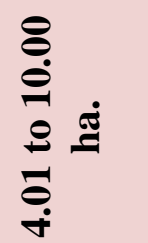 & 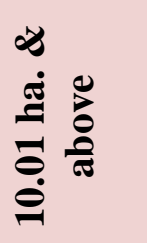 & \\
\hline 1 & Sewing & $\begin{array}{c}8 \\
(16.70)\end{array}$ & $\begin{array}{c}12 \\
(25.00)\end{array}$ & $\begin{array}{c}11 \\
(23.00)\end{array}$ & $\begin{array}{c}14 \\
(29.20)\end{array}$ & $\begin{array}{c}3 \\
(6.30)\end{array}$ & $\begin{array}{c}0 \\
(00.00)\end{array}$ & $\begin{array}{c}48 \\
(100)\end{array}$ \\
\hline 2 & Processing & $\begin{array}{c}4 \\
(14.90)\end{array}$ & $\begin{array}{c}6 \\
(22.20)\end{array}$ & $\begin{array}{c}6 \\
(22.20)\end{array}$ & $\begin{array}{c}5 \\
(18.50)\end{array}$ & $\begin{array}{c}6 \\
(22.20)\end{array}$ & $\begin{array}{c}0 \\
(00.00)\end{array}$ & $\begin{array}{c}27 \\
(100)\end{array}$ \\
\hline 3 & Agri. & $\begin{array}{c}6 \\
(14.60)\end{array}$ & $\begin{array}{c}12 \\
(29.30)\end{array}$ & $\begin{array}{c}10 \\
(24.40)\end{array}$ & $\begin{array}{c}7 \\
(17.10)\end{array}$ & $\begin{array}{c}6 \\
(14.60)\end{array}$ & $\begin{array}{c}0 \\
(00.00)\end{array}$ & $\begin{array}{c}41 \\
(100)\end{array}$ \\
\hline 4 & AHD & $\begin{array}{c}5 \\
(17.20)\end{array}$ & $\begin{array}{c}6 \\
(20.70)\end{array}$ & $\begin{array}{c}7 \\
(24.20)\end{array}$ & $\begin{array}{c}6 \\
(20.70)\end{array}$ & $\begin{array}{c}5 \\
(17.20)\end{array}$ & $\begin{array}{c}0 \\
(00.00)\end{array}$ & $\begin{array}{c}29 \\
(100)\end{array}$ \\
\hline 5 & Other business & $\begin{array}{c}6 \\
(24.00)\end{array}$ & $\begin{array}{c}7 \\
(28.00)\end{array}$ & $\begin{array}{c}6 \\
(24.00)\end{array}$ & $\begin{array}{c}5 \\
(20.00)\end{array}$ & $\begin{array}{c}1 \\
(4.00)\end{array}$ & $\begin{array}{c}0 \\
(00.00)\end{array}$ & $\begin{array}{c}25 \\
(100)\end{array}$ \\
\hline 6 & Labour & $\begin{array}{c}5 \\
(12.50)\end{array}$ & $\begin{array}{c}31 \\
(77.50)\end{array}$ & $\begin{array}{c}4 \\
(10.00)\end{array}$ & $\begin{array}{c}0 \\
(00.00)\end{array}$ & $\begin{array}{c}0 \\
(00.00)\end{array}$ & $\begin{array}{c}0 \\
(00.00)\end{array}$ & $\begin{array}{c}40 \\
(100)\end{array}$ \\
\hline & $\begin{array}{l}\text { Average } \\
\text { Ind holding }\end{array}$ & $\begin{array}{c}34 \\
(16.20)\end{array}$ & $\begin{array}{c}74 \\
(35.20)\end{array}$ & $\begin{array}{c}44 \\
(21.00)\end{array}$ & $\begin{array}{c}37 \\
(\mathbf{1 7 . 6 0})\end{array}$ & $\begin{array}{c}21 \\
(10.00)\end{array}$ & $\begin{array}{c}\mathbf{0} \\
(\mathbf{0 0 . 0 0})\end{array}$ & $\begin{array}{r}210 \\
(100)\end{array}$ \\
\hline
\end{tabular}

(Figures in the parentheses indicate percentages) 
Table.8 Distribution of the SHGs members according to their experience in SHGs enterprise

\begin{tabular}{|c|l|c|c|c|c|}
\hline \multirow{2}{*}{ Sr. No. } & \multirow{2}{*}{ Enterprise } & \multicolumn{3}{|c|}{ Category } & \multirow{2}{*}{ Total } \\
\cline { 3 - 5 } & & Low & Medium & High & \multirow{2}{*}{ (n) $)$} \\
\cline { 3 - 5 } & & Up to 2 & $\mathbf{3 ~ t o ~ 8}$ & $\mathbf{9 ~ \& ~ a b o v e ~}$ & \\
\hline $\mathbf{1}$ & Sewing & $4(8.30)$ & $\mathbf{4 4}(\mathbf{9 1 . 7 0})$ & $0(00.00)$ & $\mathbf{4 8}(\mathbf{1 0 0})$ \\
\hline $\mathbf{2}$ & Processing & $3(11.10)$ & $\mathbf{2 4}(\mathbf{8 8 . 9 0})$ & $0(00.00)$ & $\mathbf{2 7}(\mathbf{1 0 0})$ \\
\hline $\mathbf{3}$ & Agri. & $6(14.60)$ & $\mathbf{3 5}(\mathbf{8 5 . 4 0})$ & $0(00.00)$ & $\mathbf{4 1}(\mathbf{1 0 0})$ \\
\hline $\mathbf{4}$ & AHD & $5(17.20)$ & $\mathbf{2 4}(\mathbf{8 2 . 8 0})$ & $0(00.00)$ & $\mathbf{2 9}(\mathbf{1 0 0})$ \\
\hline $\mathbf{5}$ & Other business & $2(8.00)$ & $\mathbf{2 3}(\mathbf{9 2 . 0 0})$ & $0(00.00)$ & $\mathbf{2 5}(\mathbf{1 0 0})$ \\
\hline $\mathbf{6}$ & Labour & $4(10.00)$ & $\mathbf{3 6}(\mathbf{9 0 . 0 0})$ & $0(00.00)$ & $\mathbf{4 0}(\mathbf{1 0 0})$ \\
\hline \multicolumn{2}{|c|}{ Average experience } & $\mathbf{2 4}(\mathbf{1 1 . 4 0})$ & $\mathbf{1 8 6}(\mathbf{8 8 . 6 0})$ & $\mathbf{0}(00.00)$ & $\mathbf{2 1 0}(\mathbf{1 0 0})$ \\
\hline \multicolumn{2}{|c|}{ Mean $=\mathbf{4 . 9 6}$} & & S.D. $=\mathbf{3 . 4 4}$ & \\
\hline
\end{tabular}

(Figures in the parentheses indicate percentages)

Table.9 Distribution of the SHGs members according to their information seeking behaviour

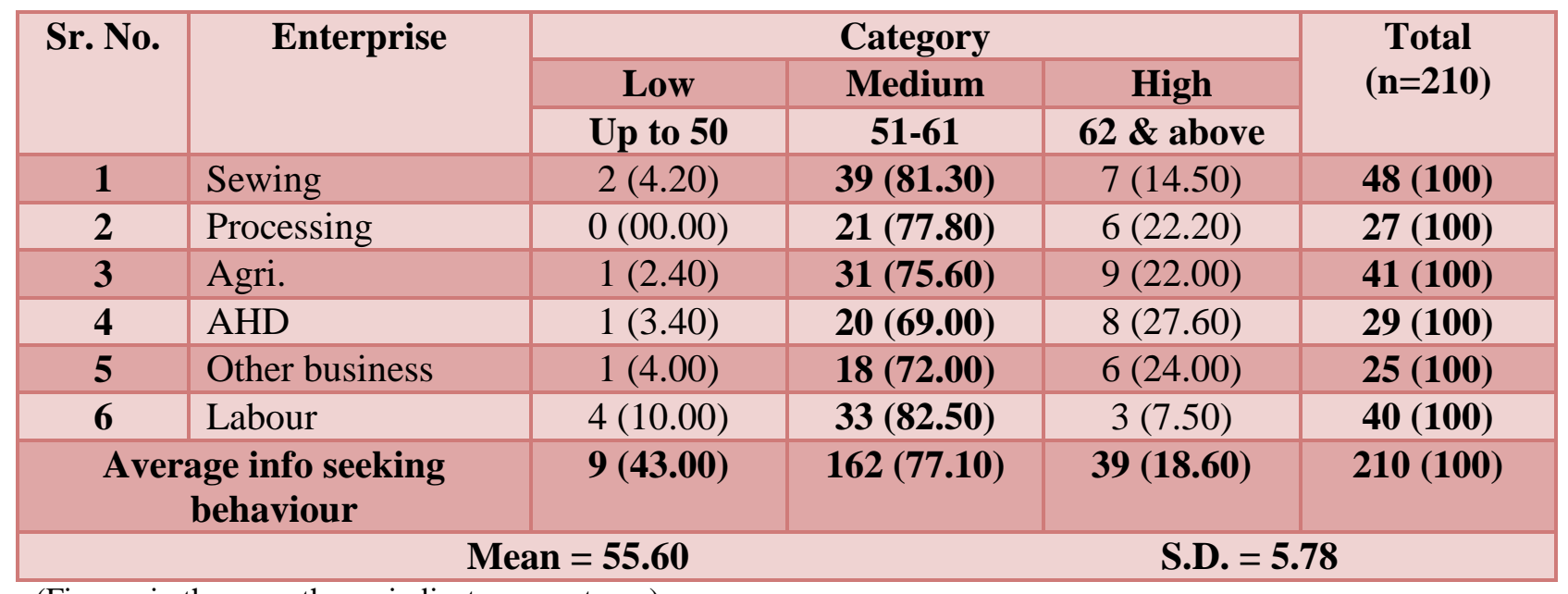

(Figures in the parentheses indicate percentages)

Table.10 Distribution of the SHGs members according to their institutional training received

\begin{tabular}{|c|l|c|c|c|c|}
\hline \multirow{2}{*}{ Sr. No. } & \multirow{2}{*}{ Enterprise } & \multicolumn{3}{|c|}{ Category } & \multirow{2}{*}{ Total $(\mathbf{n = 2 1 0})$} \\
\cline { 3 - 5 } & & Low & Medium & High & \\
\cline { 3 - 5 } & & Up to 0 & $\mathbf{1}$ & $\mathbf{2 ~ \& ~ a b o v e ~}$ & \\
\hline $\mathbf{1}$ & Sewing & $18(37.50)$ & $\mathbf{2 8}(\mathbf{5 8 . 3 0})$ & $2(4.20)$ & $\mathbf{4 8}(\mathbf{1 0 0})$ \\
\hline $\mathbf{2}$ & Processing & $10(37.00)$ & $\mathbf{1 4}(\mathbf{5 1 . 9 0})$ & $3(11.10)$ & $\mathbf{2 7}(\mathbf{1 0 0})$ \\
\hline $\mathbf{3}$ & Agri. & $14(34.10)$ & $\mathbf{2 7}(\mathbf{6 5 . 9 0})$ & $0(00.00)$ & $\mathbf{4 1}(\mathbf{1 0 0})$ \\
\hline $\mathbf{4}$ & AHD & $11(38.00)$ & $\mathbf{1 6}(\mathbf{5 5 . 1 0})$ & $2(6.90)$ & $\mathbf{2 9}(\mathbf{1 0 0})$ \\
\hline $\mathbf{5}$ & Other business & $4(16.00)$ & $\mathbf{2 1}(\mathbf{8 4 . 0 0})$ & $0(00.00)$ & $\mathbf{2 5}(\mathbf{1 0 0})$ \\
\hline $\mathbf{6}$ & Labour & $4(10.00)$ & $\mathbf{3 6}(\mathbf{7 . 5 0})$ & $0(00.00)$ & $\mathbf{4 0}(\mathbf{1 0 0})$ \\
\hline \multicolumn{2}{|c|}{ Average Training } & $\mathbf{6 1}(\mathbf{2 9 . 1 0})$ & $\mathbf{1 4 2 ( 6 7 . 6 0 )}$ & $\mathbf{7 ( 3 . 3 0 )}$ & $\mathbf{2 1 0}(\mathbf{1 0 0})$ \\
\hline \multicolumn{2}{|c|}{ Mean $=\mathbf{1 . 0 2}$} & S.D. $=\mathbf{0 . 8 2}$ \\
\hline
\end{tabular}

(Figures in the parentheses indicate percentages) 
Table.11 Distribution of the SHGs members according to their sources of motivation

\begin{tabular}{|c|c|c|c|c|c|}
\hline \multirow{3}{*}{$\begin{array}{l}\text { Sr. } \\
\text { No. }\end{array}$} & \multirow[t]{3}{*}{ Enterprise } & \multicolumn{3}{|c|}{ Category } & \multirow[t]{3}{*}{ Total $(n=210)$} \\
\hline & & Low & Medium & High & \\
\hline & & Up to 1 & 2 & 3 \& above & \\
\hline 1 & Sewing & $1(2.00)$ & 47 (98.00) & $0(00.00)$ & $48(100)$ \\
\hline 2 & Processing & $2(7.40)$ & $25(92.60)$ & $0(00.00)$ & $27(100)$ \\
\hline 3 & Agri. & $0(00.00)$ & $41(100)$ & $0(00.00)$ & $41(100)$ \\
\hline 4 & AHD & $1(3.40)$ & $28(96.60)$ & $0(00.00)$ & $29(100)$ \\
\hline 5 & Other business & $0(00.00)$ & $25(100)$ & $0(00.00)$ & $25(100)$ \\
\hline 6 & Labour & $0(00.00)$ & $40(100)$ & $0(00.00)$ & 40 (100) \\
\hline \multicolumn{2}{|c|}{$\begin{array}{c}\text { Average source of } \\
\text { motivation }\end{array}$} & $4(2.00)$ & $206(98.00)$ & $\mathbf{0}(00.00)$ & $210(100)$ \\
\hline \multicolumn{4}{|c|}{ Mean $=1.63$} & S.D. = & \\
\hline
\end{tabular}

(Figures in the parentheses indicate percentages)

\section{Annual income}

The main objective of self help groups is to provide employment opportunities to rural poor in order to improve their economic conditions. SHGs helped them to cross below poverty line by increasing their income level. It is interesting to note that majority of SHGs members was coming under medium income groups after the engaged in different enterprise.

It was observed from table 5 that, two third $(68.10 \%)$ of the SHGs members had medium annual income i.e. (Rs. 25219/- to Rs. 57123/-). It was also found that majority 91.70 per cent of the SHGs members of having medium annual income were engaged in sewing operation. These findings were further strengthened by the results reported by Mankar et al., (2013), Bhandari (2014), Khandave and Suryawanshi (2015) and Dhulgand (2020).

\section{Occupation}

It was observed from table 6 that, majority $(60.50 \%)$ of the SHGs members were engaged in main + subsidiary occupation. It was also observed that majority 88.00 per cent of the SHGs members who were engaged in both main+ subsidiary occupation mostly in other business. The selected SHGs members were practicing in multiple enterprises due to being ancestral traditional occupation and also due to gain in profit from number of enterprise. These findings were further strengthened by the results reported by Peerzade and Parande (2005).

\section{Land holding}

Size of land holding largely determines both economic as well as social status of the family in the rural areas and also decides the extent of their dependence on agriculture also other allied occupation. The data regarding the land owned by the families of the SHGs members. It was revealed from table 7 that, at overall level, majority 35.20 per cent of the SHGs members had 'marginal' land holding. It was also noticed that majority 77.50 per cent of the SHGs members having marginal land holding were labours. It can be concluded from these findings that the SHGs members were having less land for cultivation, which might have been put under different nonremunerative crops. The probable reason might be that the land holding is reducing day by day due to fragmentation of land which in 
turn is due to division of family also not being cultivated and have resulted in low yield than their family requirements. Hence, for earning of additional income to run their day to day life, they might have joined SHGs. The findings of the present study were in agreement with the findings of Kulkarni (2003).

\section{Experience in SHGs enterprise}

Individuals with more experience in their respective enterprise are likely to perform variety of works efficiently. It was therefore, thought that members with more experience might have been contributing more for development of their SHGs enterprise. It was observed from table 8 that, at overall level majority 88.60 per cent of the SHGs members had 'medium' experience in their respective enterprise. At overall level, the average experience of the members of SHGs was about 3-8 years. The enterprise wise experience of the SHGs members reported highest in other business $(92.00 \%)$. It can be concluded that at overall level, majority of the SHG members had low to medium that is three to eight years of experience in their enterprise. Majority of members from other business group had high experience because those groups were formed by the individuals who had different work as their caste occupation. Further, majority of the members from processing group had up to three years of experience in fruit processing, meaning thereby that the activity was new for them. The findings pertaining to age of the group and experience of the members in the respective enterprise made it clear that majority of the members did not have earlier experience in the concerned enterprises. This underlines the importance of imparting training to the members in their group's enterprise, so that they would be able to manage the enterprises efficiently and profitably. The findings were in line with findings of Aimable (2011), Fazely (2016) and Murai (2016).

\section{Information seeking behaviour}

Information seeking behaviour influences an individual by having more opportunities to come in contact with different kind of sources. This helps in securing more information through various sources. It was therefore, thought that information seeking behaviour of the members of self help groups might influence their performance in the enterprise. It was revealed that, at overall level, majority 53.11 per cent of the SHGs members had 'medium' information seeking behaviour. The majority score of information seeking behaviour at overall level was 82.50 per cent of the SHGs members were labours. The findings of the present study were in line with the findings of Sarvankumar (2000) and Ganeshmurthy et al., (2004) (Table 9).

\section{Institutional training received}

Training is a very important input, especially in the context of low level of literacy and poor skills among the rural poor. SHGs members were being trained by various agencies on a whole range of activities. It was revealed from table 10 that, majority 67.60 per cent of the SHGs members were in 'medium' category of training received as well as it was also noticed that majority 84.00 per cent of the SHGs members having medium training were engaged in other business. It could be concluded that most of the SHGs members had undergone training, but only to a lower degree. The bank officials, extension agencies and NGOs might have organized trainings for the SHGs members in order to impart them knowledge and skill for day to day management of the SHGs and their enterprise. These findings also suggest that there should be need for motivating the SHGs members to participate in the training about their enterprises. The findings of the present 
study were in line with the findings of (2007), Aimable (2011), Gopika (2014) and Fazely (2016).

\section{Sources of motivation}

In the present study, motivation was considered as a process of creating an urge or stimulation towards participation in self help group by some sources. One may be motivated by, several sources for participation in SHGs. Hence, the nature and number of sources who had motivated the members to participate in self help group were studied, and the source was worked out. The date in table 11 indicated that, majority 98.00 per cent of the SHGs members had utilized 'medium' as sources of motivation for participation in SHGs and it was also noticed that 100 per cent of the SHGs members utilized medium sources of motivation were engaged in agriculture sector. Therefore, it is inferred that most of the SHGs members had moderate score of sources of motivation to participate in SHGs. This might be attributed to the nature of sources that motivated the members and also their number. It seems that the members were motivated mostly by a few number of localite or local cosmopolite sources to participate in the SHGs.

It means majority of the members were motivated to participate in the SHGs either by the members of their family and community, or by the local professional workers of different government, semi-government and non-government organizations. The outside sources seem to have played least role in motivating the members to participate in the SHGs. Thus, the motivational efforts seem to be confined at local level. Though, this is natural and logical, the need for motivational efforts by the higher officials and non officials from outside the social system becomes evident. Secondly, training to the motivators in various techniques of motivation may help in strengthening their work. The results of the study conducted by Kulkarni (2003), Adsul (2004) and Top are (2006) are in line with the findings of the present study.

It was concluded that many of the self help group's women have been benefited after actively joining in the self help groups and also through microfinance. As SHGs members believe that age is just a number for them and they can startup any kind of enterprise for the upliftment of their self and their families. So it's very important to be educated, develop the information seeking behaviour, get trained in respective field to perform efficiently and get always be motivated to do different things to get changeover of their life.

\section{References}

Adsul, Archana H. (2004). Case studies of self-help units under SGSY of Gramin Mahila Va Balak Vikas Mandal of Bank of Maharashtra in Pune district. M.Sc. (Agri.) Thesis (Maharashtra), Mahatma Phule Krishi Vidyapeeth, Rahuri.

Adsul, G. B. (2016). Socio-economic impact of National Horticulture Mission on its beneficiaries in Marathwada region. Ph.D. (Agri.) Thesis, Vasantrao Naik Marathwada Krishi Vidyapeeth, Parbhani (Maharashtra).

Aimable, R. (2011). An analysis of Job perception and Job performance in the University of Agricultural Sciences, Bangalore, M. Sc. (Agri.), Thesis, (University of Agricultural Sciences, Bangalore.

Bhandari, S.D. (2014). Impact of Mahatma Gandhi national rural employment guarantee act on the beneficiaries. M.Sc. (Agri.) Thesis, Vasantrao Naik Marathwada Krishi Vidyapeeth, Parbhani (Maharashtra).

Dhulgand, V. G. (2020). Socio-economic 
impact of Mahatma Gandhi National Rural Employment Guarantee act on its beneficiaries in Marathwada region. Ph.D. (Agri.) Thesis, Vasantrao Naik Marathwada Krishi Vidyapeeth, Parbhani (Maharashtra).

Fazely, A.S. (2016). (Agri), Thesis A Study on job perception, job performance and job satisfaction of teachers of state Agricultural Universities in Karnataka

Ganesamurthy, V.S., M.K. Radha Krishnan., S. Bhuvaneswari and Ganesan, A. (2004). A study on thrift and credit utilization pattern of SHG in Lakshmi Vikas Bank, Suriyampalayam Branch, Erode. Indian Journal of Marketing 34(1): 12-16.

Gopika, M. H. (2014). Study on Participation in Decision Making, Job Satisfaction and Job Performance of Assistant Horticulture Officers, M. Sc.(Agri.), Thesis (Dharwad), University of Agricultural Sciences, Bangalore.

Hasan, K., Abu Habib, Md. Abdullah, D. Bhattacharjee and S.I. Afrad (2016). Impact of alternative wetting and drying techniques of Rice production in the drought prone areas of Bangladesh. Indian Res. J. Ext. Edu. 16 (1), pp: 3948.

Jadhav, A. R. (2018). Socio-economic, psychological and situational causes of farmers suicides in Osmanabad district. M.Sc. (Agri.) Thesis, Vasantrao Naik Marathwada Krishi Vidyapeeth, Parbhani (Maharashtra.)

Khandave, S.R. and P.S. Suryawanshi (2015). Impact of National Horticulture Mission on beneficiaries. Journal of Agriculture Research Technology 40 (2), pp: 348350.

Krishnakumar, G. (2002). A study on farm women participation in the TANWA self help groups.M.Sc. (Agri) Thesis, Tamil Nadu Agricultural University, Coimbatore
Kulkarni, N.M. (2003). A study of beneficiary women of self help group organized by Jana Parbodhni with special referee to their socio-economic developme;nt. M.Sc. (Agri.) Thesis, Mahatma Phule Krishi Vidyapeeth, Rahuri (Maharashtra).

Lairinliana, Joseph and Kanagarai, Easwaran (2006). SHGs and tribal development in Mizoram. Kurukshetra 54(3): 37 - 48.

Lathkar Malhar, (2009). Importance Of Self Help Groups In Rural Development

Mankar, D.M., P.P. Wankhade and Shambharkar, Y.B. (2013). Impact of National Horticulture Mission on its beneficiaries. International Journal of Extn. Edu. (9), pp: 72-80.

Murai, A.M. (2016). Job performance and job satisfaction of academic staff of Vasantrao Naik Marathwada Krishi Vidyapeeth, Parbhani.

Pattanaik, B.K. (2009). National Rural Employment Guarantee Scheme (MREGS): Some preliminary findings from Hoshiarpur district. Kurukshetra 57(6), pp: 35-40.

Peerzade, S.A. and Prema Parande (2005). Employment of women: A study. Kurukshetra, 54(1): 7-11.

Raut, M.A. (2018). Attitude of beneficiaries towards Mahatma Gandhi National Rural Employment Guarantee Act (MGNREGA) M.Sc. Agri.Thesis Dr. Panjabrao Deshmukh Krishi Vidyapeeth, Akola (Maharashtra).

Rewale, Rajani A. (2003). A study on women's self-help groups engaged in agricultural activities in Ratnagiri district. M.Sc. (Agri.) Thesis, Dr. Balasaheb Sawant Konkan Krishi Vidyapeeth, Dapoli.

Saravanakumar, M.M. (2000). Impact of TANWA in the empowerment of farm women, M.sc. (Agri.) Thesis, Tamil Nadu Agricultural University, Coimbatore. 
Singh, U.B., Singh Himmat and Singh Gurnam (2001). Role of SHGs in the Shiwalik Foot Hills of Haryana. Indian Journal of Agricultural Economics 56(3): 481-482.

Sucharita, S. and Bishnoi I. (2019). Socio Economic Status of SHG members : A study in Ranapur block of Odisha Socio Economic Status of SHG members : A study in Ranapur block of Odisha.
Research Review International Journal of Multidisciplinary, Vol. 4(1), January, 6-9.

Topare, Dipali (2006). Impact on employment generation and income of women members of self help groups. M.Sc. (Agri). Thesis, Dr. Balasaheb Sawant Konkan Krishi Vidyapeeth, Dapoli.

\section{How to cite this article:}

Ramadevi G. Nair, R. P. Kadam and Lad, A. S. 2021. Study the Profile of the SHGS Members Engaged in Agriculture and Allied Enterprises. Int.J.Curr.Microbiol.App.Sci. 10(02): 34963507. doi: https://doi.org/10.20546/ijcmas.2021.1002.385 